\title{
Galaxies at high redshifts: where are the progenitors of today's dwarf ellipticals?
}

\author{
Max Pettini \\ Institute of Astronomy, University of Cambridge, Madingley Road, \\ Cambridge CB3 0HA, England \\ email: pettini@ast.cam.ac.uk
}

\begin{abstract}
The epochs when many dwarf ellipticals were forming their stellar populations have recently become accessible to direct observation, as surveys at $z=1-5$ have now uncovered thousands of galaxies. I briefly review the properties of various categories of high redshift galaxies in search of clues for possible links to today's galaxy populations. While much of the emphasis up to now has been on massive elliptical galaxies, with several groups claiming to have identified their progenitors with a variety of different techniques, dwarf galaxies have generally been overlooked - no doubt because the observational difficulties at high redshift are still considerable. Based on available data, it is possible that at least some of the 'Ly $\alpha$ emitters' and the damped Ly $\alpha$ systems may be on evolutionary paths which will transform them into the galaxies which are the subject of this meeting.
\end{abstract}

\section{Introduction}

I should like to echo Eva Grebel's words in her excellent review which opened this Colloquium and emphasized the importance of local studies as the key to interpreting the increasingly detailed picture of the high redshift universe which has been emerging in recent years. The wealth of data being gathered in galaxy surveys which stretch from the present-day universe (e.g. Croton et al. 2005), through intermediate (e.g. Madgwick et al. 2003; Abraham et al. 2004) and high (e.g. Steidel et al. 2004, 2005) redshifts, to the epoch when the universe became reionised (e.g. Bunker et al. 2004) is truly staggering. The thread which is common to all of these efforts is the desire to follow the evolution of the Hubble sequence of galaxies backwards in time so as to identify the progenitors of today's elliptical and spiral galaxies, and thereby throw light on the physical processes driving their formation and which must be at the root of their morphological differences.

Of relevance to the theme of this meeting is therefore the question "Have we found any clues to the origin of dwarf elliptical galaxies in the high-redshift galaxy population?" By their very nature, dwarf galaxies are obviously the most difficult to see in any magnitudelimited survey of the distant universe. Nevertheless, it is worthwhile confronting the properties of the galaxies being targeted by high redshift surveys with those of the dwarf ellipticals whose stellar populations, as we have learnt from Eva's work, in many cases formed at redshifts $z=2-6$, that is at the same early cosmic epochs now accessible to direct observation.

I shall address this question for the two classes of high redshift galaxies (without active galactic nuclei) which have been most extensively studied up to now: the Lyman break galaxies and the damped Lyman $\alpha$ systems. 

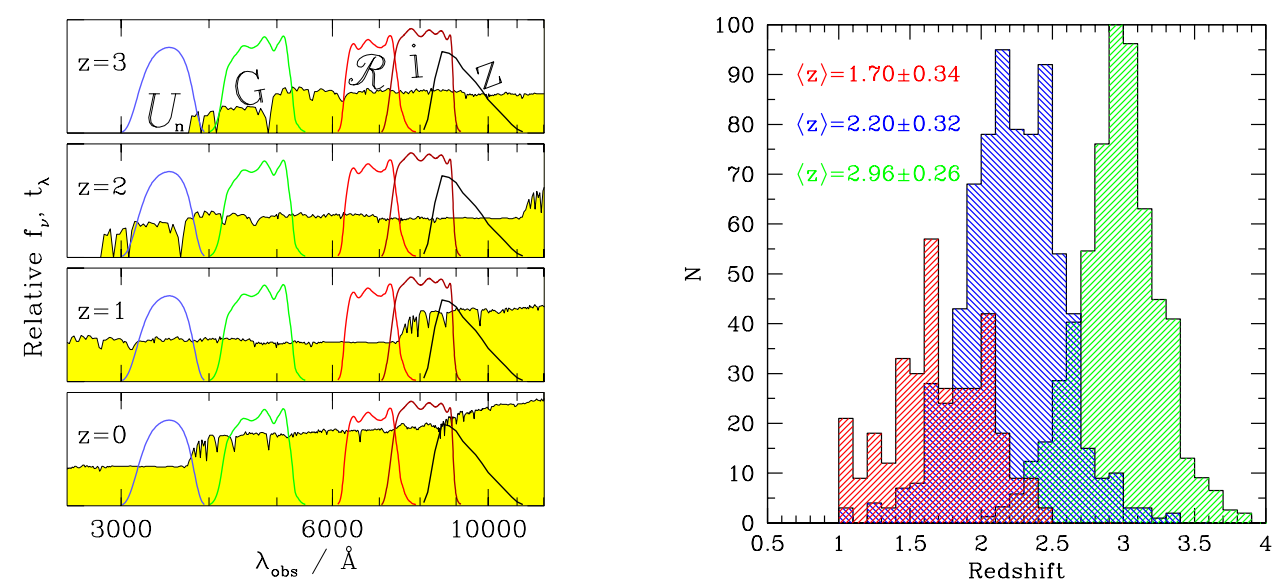

Figure 1. Left Panel (reproduced from Adelberger et al. 2004): The $U_{n} G \mathcal{R} i z$ filter passbands straddle different spectral features at different redshifts in the spectral energy distribution of a typical star-forming galaxy. Consequently, galaxies can be photometrically preselected within reasonably well-defined redshift intervals which are well separated on a colour-colour plane, as shown by the redshift distributions of spectroscopically confirmed galaxies from the surveys by Steidel et al. 2004 (shown in the Right Panel).

\section{Star-forming galaxies at $z=1.5-3.5$}

Lyman break galaxies are just one example of UV-bright - and therefore star-forminggalaxies selected within a predetermined redshift interval by the application of appropriate broad-band photometric criteria. The original $U_{n} G \mathcal{R}$-based colours of Steidel, Pettini \& Hamilton (1995) have more recently been 'tuned' (see Adelberger et al. 2004) to select galaxies at mean redshifts $\langle z\rangle=3.0$ (LBGs), 2.2 (BX galaxies) and 1.7 (BM galaxies), in each case with a redshift dispersion of about 0.3 (see Figure 1 ).

The $B z K$ criteria of Daddi et al. (2004) can be used to select either galaxies which overlap significantly with the BX/BM galaxies of Steidel et al. (2004) or, by focusing on a different portion of the $B z K$ colour-colour plane, galaxies which by virtue of their red optical-to-infrared colour must be either passively evolving or heavily reddened. Similarly red galaxies are targeted by the FIRES survey of Franx et al. (2003). The way these different searches overlap and complement each other has recently been assessed in a comprehensive study by Reddy et al. (2005). All these populations of high redshift galaxies span a wide range in luminosity (see Adelberger \& Steidel 2000; Shapley et al. 2001), and it is the ones at the brighter end of the distribution, with $L \geqslant L^{*}$, which have naturally been the subject of most spectroscopic studies so far.

There are now several lines of evidence that lead us to associate these bright galaxies with the progenitors of today's massive ellipticals and bulges (an interpretation favoured by nearly all of the works cited in the preceding paragraph), rather than with low mass galaxies briefly lit up by a short-lived starburst episode. These lines of evidence include:

Stellar masses. Analysis of the spectral energy distributions of BX galaxies from the rest-frame UV to the rest-frame near-IR (now possible by combining ground-based deep imaging in the optical and near-IR with IR multi-band data from the Spitzer satellite) indicates assembled stellar masses in the range $M_{*}=10^{9.5}-10^{11.5} M_{\odot}$, with a median value $\left\langle\log \left(M_{*} / M_{\odot}\right)\right\rangle=10.3 \pm 0.5$ (see Figure 2 ). 

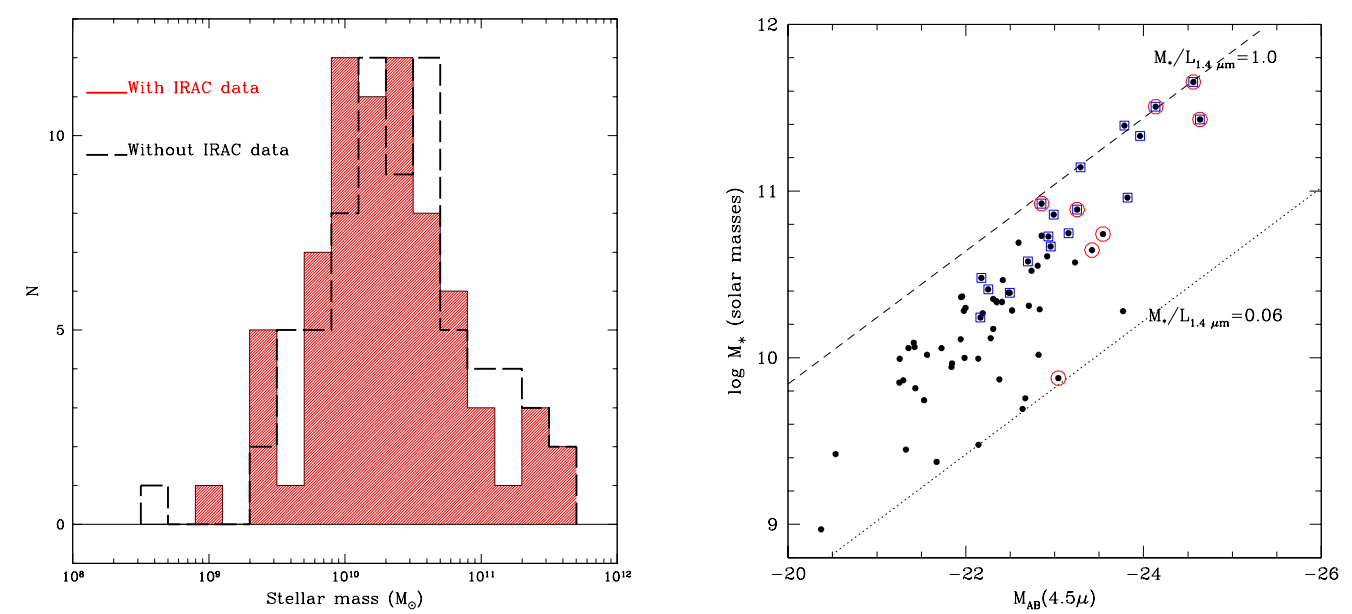

Figure 2. Stellar masses of BX galaxies in the field of the QSO HS1700+643 (for which Spitzer IRAC $3.6-8.0 \mu \mathrm{m}$ photometry is available). In the right-hand panel, the stellar masses are plotted versus the absolute magnitude at $4.5 \mu \mathrm{m}$, equivalent to rest-frame $1.4 \mu \mathrm{m}$ luminosity at the mean $\langle z\rangle=2.2$ of these galaxies. It can be seen that even in the near-IR the mass-to-light ratio of these galaxies spans a factor of $\sim 15$. Evidently, the most massive galaxies had already attained values comparable to those of present-day galaxies, while more typically $(M / L)_{1.4 \mu m} \simeq 0.4$, a factor of $\sim 2.5$ smaller. Circles indicate galaxies brighter than $K_{s}=20.0$, while open squares denote red galaxies with $\mathcal{R}-K_{s}>3.5$. (Figures reproduced from Shapley et al. 2005)

Clustering properties. These galaxies already exhibit a high degree of clustering by $z=2-$ 3 , with correlation lengths $r_{0} \simeq(4 \pm 0.5) h^{-1}$ comoving Mpc (Adelberger et al. 2004). The brightest galaxies, with $K_{s}<20$, are more strongly clustered with $r_{0}=(10 \pm 3) h^{-1} \mathrm{Mpc}$ (Adelberger et al. 2005; Daddi et al. 2004). In $\Lambda$ CDM N-body simulations (e.g. Frenk et al. $2000)$, this is the degree of clustering exhibited by massive halos, with $\log \left(M_{\mathrm{DM}} / M_{\odot}\right) \sim$ 11-12, which by the present day evolve to have number densities and correlation lengths typical of elliptical galaxies (see Figure 3).

Metallicities. A variety of techniques - among which the ratio of the $[\mathrm{N}$ II] $]$ 6583 and H $\alpha$ emission lines has had the widest application so far (see Figure 4) - give a concordant picture showing that these galaxies had already reached solar, or near-solar, abundances at $z=2-3$. While they are undoubtedly bright for their metallicity compared to the present-day metallicity-luminosity relation (another indication of their low $M / L$ ratios), it is hard to conceive of a plausible scenario by which these galaxies would evolve into today's metal-poor dwarf galaxies.

Presumably, and not surprisingly, the progenitors of today's dwarf galaxies are to be found among objects fainter than $L^{*}$ in the luminosity function of high redshift galaxies. Up to now these candidates have been little studied spectroscopically, as they are beyond the reach of current instrumentation, except for the fraction which shows a strong Lyman $\alpha$ emission line (e.g. Dawson et al. 2004; Hu et al. 2004). Ly $\alpha$ emitters have been identified in large numbers at redshifts up to $z=6.6$ (Taniguchi et al. 2005), and among them there may well be galaxies on an evolutionary path which will lead them to become today's dwarf ellipticals.

\section{Damped Lyman $\alpha$ absorption systems}

With column densities of neutral hydrogen in excess of $N(\mathrm{H} \mathrm{I})=2 \times 10^{20} \mathrm{~cm}^{-2}$, DLAs account for most of the neutral gas seen in absorption along random sight-lines to 

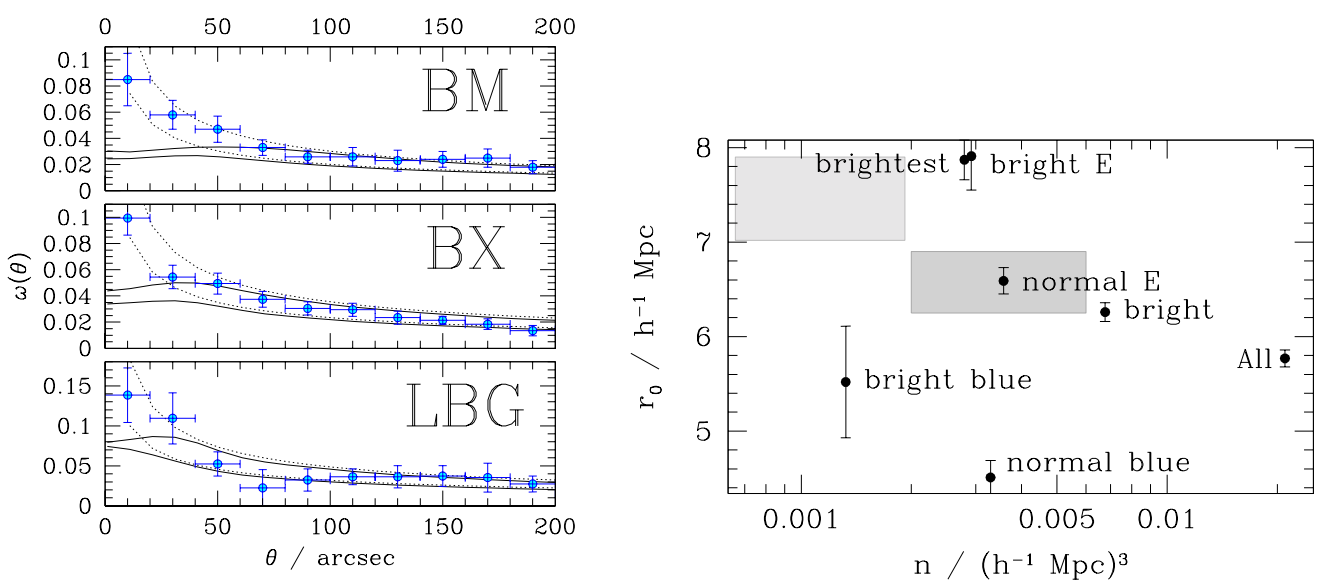

Figure 3. Left Panel: The measured angular correlation functions of LBG, BX, and BM galaxies (points with error bars) match those of dark matter halos with masses in the range $\log \left(M_{\mathrm{DM}} / M_{\odot}\right) \sim 11-12$ in N-body simulations such as that released by the Virgo consortium (Frenk et al. 2000) and indicated by the continuous lines. Right Panel: Following the evolution of these massive halos forward in time shows that, by $z=0.2$, LBG, BX, and BM galaxies will have attained correlation lengths $r_{0} \simeq 7 h^{-1} \mathrm{Mpc}$, similar to those of elliptical galaxies. The shaded rectangles show the ranges in the values of $r_{0}$ and $n$ (number density per unit volume) expected for the descendents of 'typical' (near the centre of the plot), and the brighter $\left(L>L^{*}\right.$; top left-hand corner) $z=3$ Lyman break galaxies. The points with error bars are values of $r_{0}$ and $n$ measured by Budavari et al. (2003) for various samples of galaxies from the Sloan Digital Sky Survey, as labelled. (Figures reproduced from Adelberger et al. 2004).
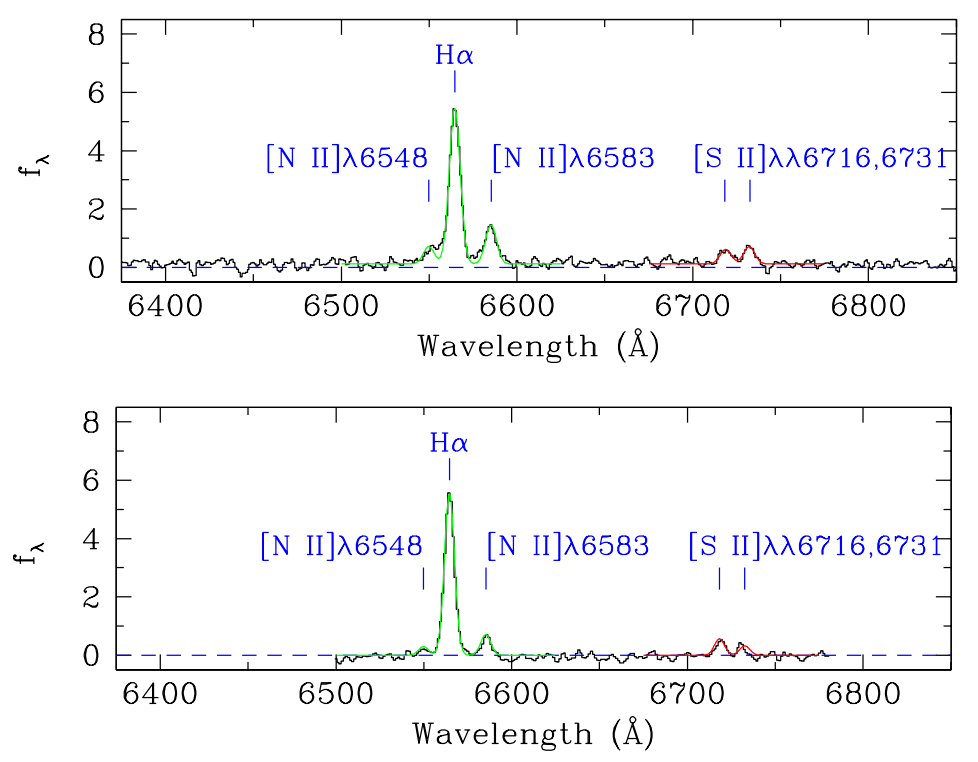

Figure 4. Upper Panel: Composite spectrum of $21 \mathrm{BX}$ galaxies brighter than $K_{s}=20$ obtained with NIRSPEC on the Keck II telescope (Erb et al. 2005). Lower Panel: Composite spectrum of 66 BX galaxies fainter than $K_{s}=20$. Using the calibration by Pettini \& Pagel (2004), the measured [N II] $\lambda 6583 / \mathrm{H} \alpha$ ratios imply approximately solar metallicities for the $K_{s}<20$ galaxies, and about half-solar for the fainter ones. 

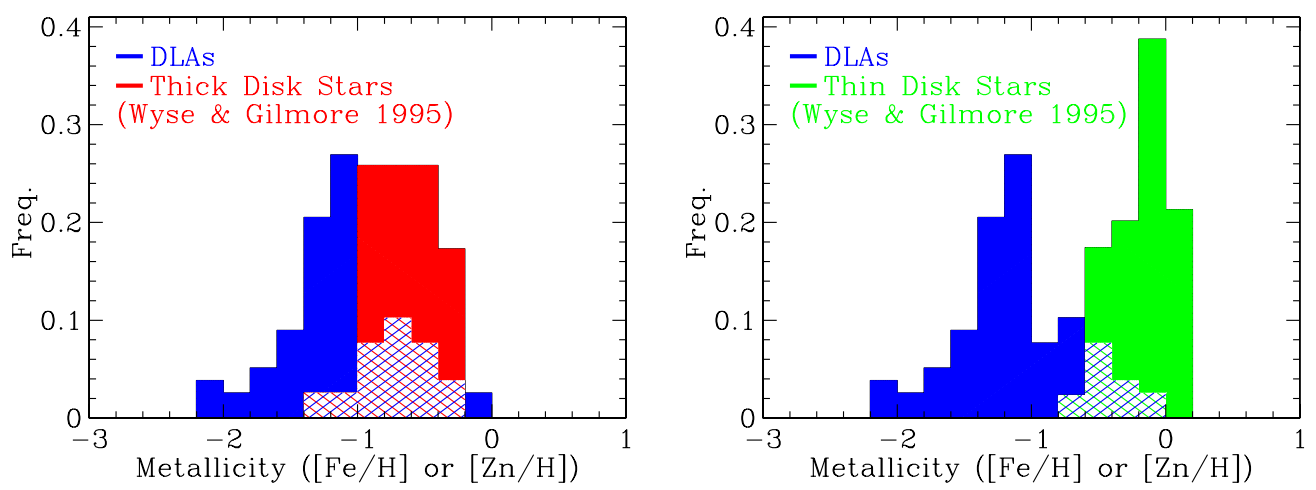

Figure 5. Metallicity distributions of DLAs and Milky Way thick (left panel) and thin (right panel) disk stars from the work by Akerman et al. (2005). The metallicity is measured from $\mathrm{Zn}$ in DLAs and Fe in Galactic stars. Approximately one half of the DLA measurements of $[\mathrm{Zn} / \mathrm{H}]$ are upper limits; they have been included in the histograms as if they were detections.

distant QSOs. After two decades of extensive observational work, we know many of their properties in exquisite detail - such is the power of QSO absorption line spectroscopy, but somehow this makes it all the more frustrating that their connection to present-day galaxies is still a matter of debate. H I surveys of the local universe now seem to indicate that a wide range of galaxy luminosities (from $M_{B} \simeq-21$ to -15 ) and morphological types can contribute to the cross-section for DLA absorption (Zwaan et al. 2005). At higher redshifts, $z=1.5-3.5$, it is unclear how DLAs relate to other galaxy populations. The working hypothesis since the early days of this subject has been that DLAs are the progenitors of spiral galaxies, observed at a time when most of their baryons were in the interstellar medium rather than in stars, but this picture may be at odds with theoretical ideas of a slow build-up of disks over most of the Hubble time (e.g. Naab \& Ostriker 2005). The possibility that at least some high- $z$ DLAs may be associated with dwarf galaxies is, on the other hand, supported by several observational results:

Luminosities. The galaxies which host $z \sim 3$ DLAs are generally fainter than $L^{*}$ LBGs (Cooke et al. 2005).

Metallicities. DLAs are metal-poor, with typical abundances between 1/10 and 1/100 of solar (Akerman et al. 2005). As can be seen from Figure 5, the metallicity distribution of DLAs peaks at lower values than those of Milky Way thick and thin disk stars, although there is some overlap, particularly with the thick disk. The difficulty here for the spiral galaxy interpretation of DLAs is that the period of time during which the Milky Way disk was as metal-poor as a typical DLA was probably short-lived, as evidenced by the near-flat age-metallicity relation of its stars. A possible way out may be a scenario where the (relatively) metal-rich, star-forming, inner disk is surrounded by a larger (thick) disk of mostly unprocessed gas, as proposed by Wolfe (2005). More generally, steep radial abundance gradients in young disks (Naab \& Ostriker 2005) may be the explanation for the low metallicity of most DLAs.

Element ratios. There has been much discussion of recent findings that stars in dwarf spheroidals of the Local Group may have lower $\alpha /$ Fe ratios than Galactic halo stars of similar $\mathrm{Fe} / \mathrm{H}$, and of what the implications of such differences might be for hierarchical models in which the halo was assembled by the mergers of many such sub-units (see, 

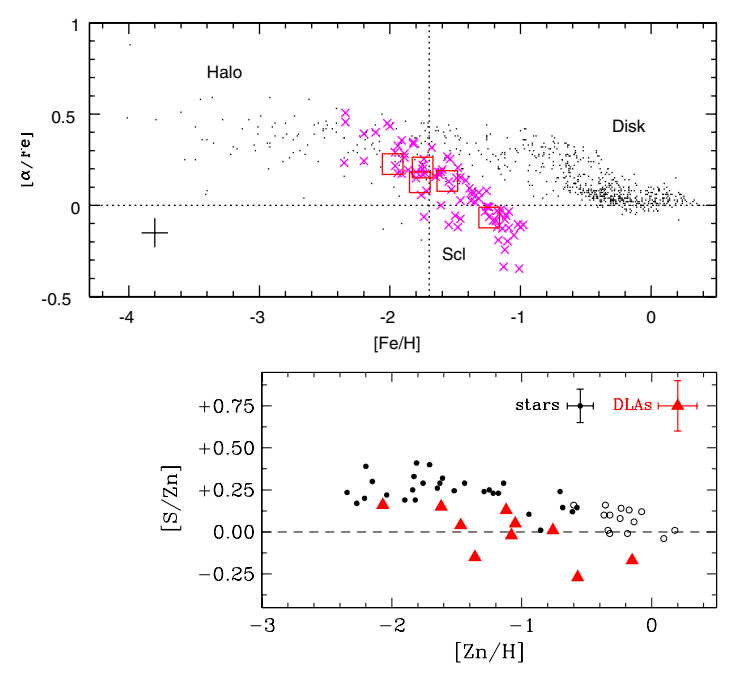

Figure 6. Upper Panel: $\alpha$-element/Fe ratios of individual stars in the Sculptor dwarf spheroidal galaxy (crosses and large open squares) compared with the values measured in Milky Way stars (diagram reproduced from Tolstoy 2005). Lower Panel: Ratio of S (an $\alpha$-capture element) to $\mathrm{Zn}$ (an Fe-peak element) in Galactic stars and DLAs (reproduced from Nissen et al. 2004). While some Sculptor stars and some DLAs conform to the well-known $\alpha$-element enhancement typical of the metal-poor Galactic halo, in general there appears to be a wider spread of $[\alpha / \mathrm{Fe}]$ values in the former environments compared to the latter. Such differences bring into question the working hypotheses that: (a) the Galactic halo was assembled by many smaller sub-units similar to the Local Group dwarf galaxies which survive today, and (b) DLAs arise in the progenitors of disk galaxies like the Milky Way.

for example, Eline Tolstoy's contribution to this volume). It is thus intriguing that even from this point of view DLAs resemble more closely dwarf galaxies than the Milky Way (see Figure 6), although the number of DLAs in which the ratio $\alpha /$ Fe-peak has been measured so far is still small.

ISM temperature. By combining values of $N(\mathrm{H} \mathrm{I})$ measured from the damping wings of the Ly $\alpha$ absorption line with the optical depth in $21 \mathrm{~cm}$ absorption, spin temperatures (or upper limits) have now been determined for 28 DLAs lying in front of radio-loud QSOs (see Figure 7). It is intriguing that at $z>2$ only high spin temperatures have been measured so far, whereas at lower redshifts both high and low values of $T_{s}$ are encountered. Furthermore, all DLAs with low $T_{s}$ have been identified with spiral galaxies, while most of the low- $z$, high $T_{s}$, DLAs are associated with $L \lesssim 0.1 L^{*}$ galaxies. If we are not being misled by the different spatial scales probed by absorption and emission line measurements, this shift in the distribution of ISM temperatures with redshift could be taken as evidence that the population of DLA absorbers is dominated by dwarf galaxies at high $z$, and that it evolves at lower redshifts to include more massive, metal-rich spirals. Presumably, the high values of $T_{s}$ of most DLAs are just another manifestation of their low metallicities, since cooling is via heavy element atomic transitions. Thus, if we could clarify why most DLAs are metal-poor at an epoch when the Milky Way disk had already been enriched to $Z \sim 1 / 3 Z_{\odot}$, and what this means for the DLA-spiral galaxy connection, we would also solve the riddle posed by their high ISM temperatures.

Of course, there are two fundamental differences between DLAs and dwarf ellipticals: DLAs are gas-rich and are large. Radii of order $10 \mathrm{kpc}$ are indicated by: (a) the frequency 


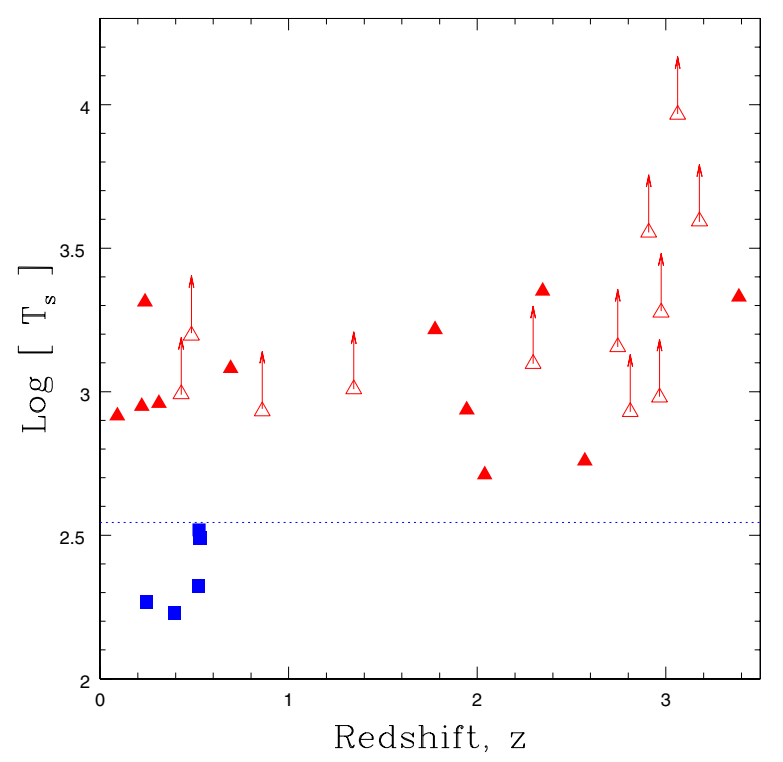

Figure 7. Spin temperatures of DLAs as a function of redshift (Kanekar \& Chengalur 2003; Kanekar et al. 2005). Squares represent DLAs identified with spiral galaxies. Filled triangles show other detections of $21 \mathrm{~cm}$ absorption, open triangles are $3 \sigma$ lower limits to the spin temperature. The horizontal dashed line indicates $T_{s}=350 \mathrm{~K}$.

with which a random sight-line to a background QSO intersects a DLA at $z=2-3$ (albeit under the unrealistic assumption of a non-evolving galaxy luminosity function, e.g. Steidel 1993), and (b) the typical impact parameters encountered at $z<1$ (which however only refer to the DLA host galaxies which have been successfully imaged; Zwaan et al. 2005). What is of interest in the context of the present discussion is whether some DLAs could turn into dwarf ellipticals if they lost their gaseous reservoirs early on in their evolutionary history. Plausible local examples of this scenario do exist (see Liese van Zee's contribution to this volume), but it remains to be established whether these rare cases were in fact more common at high redshift.

\section{Conclusions}

While large-scale surveys at a variety of wavelengths are providing an increasingly detailed picture of the high redshift universe, the connection between its denizens and today's morphological classes of galaxies has yet to be established with any degree of confidence. Many of the properties of luminous star-forming galaxies at $z=1.5$ to 3.5 - particularly their high stellar masses, metallicities and clustering lengths - point to today's massive ellipticals and bulges as their likely descendants. Presumably the high redshift counterparts of today's dwarf ellipticals are to be found among galaxies fainter than $L^{*}$. In this faint regime, we know too little as yet about the 'Ly $\alpha$ emitters' to draw meaningful comparisons, but we have identified several properties of the damped Ly $\alpha$ systems which are suggestive of a possible link, at least for some of the DLAs. These are of course only the first steps. Tracing back the evolutionary history of dwarf galaxies, whether ellipticals, irregulars, or Local Group spheroidals, still relies on the 
clues provided by their stellar populations - as the title of this colloquium implies, this is the realm of 'near-field cosmology'.

\section{Acknowledgements}

I should like the thank the organisers of this stimulating and well planned Colloquium, and the International Astronomical Union for the award of a travel grant. I am grateful to Chris Akerman and Nissim Kanekar for permission to show data in advance of publication.

\section{References}

Abraham, R.G. et al. 2004, AJ 127, 2455

Adelberger, K.L., Erb, D.K., Steidel, C.C., Reddy, N.A., Pettini, M. \& Shapley, A.E. 2005, ApJ 620, L75

Adelberger, K.L. \& Steidel, C.C. 2000, ApJ 544, 218

Adelberger, K.L., Steidel, C.C., Shapley, A.E., Hunt, M.P., Erb, D.K., Reddy, N.A. \& Pettini, M. 2004, ApJ 607, 226

Akerman, C.J., Ellison, S.L., Pettini, M. \& Steidel, C.C. 2005, $A \& A$ in press

Budavari, T. et al. 2003, ApJ 595, 59

Bunker, A.J., Stanway, E.R., Ellis, R.S. \& McMahon, R.G. 2004, MNRAS 355, 374

Cooke, J., Wolfe, A.M., Prochaska, J.X. \& Gawiser, E. 2005, ApJ 621, 596

Croton, D.J. et al. 2005, MNRAS 356, 1155

Daddi, E., Cimatti, A., Renzini, A., Fontana, A., Mignoli, M., Pozzetti, L., Tozzi, P. \& Zamorani, G. 2004, ApJ 617, 746

Dawson, S. et al. 2004, ApJ 617, 707

Erb, D.K. et al. 2005, in preparation

Franx, M. et al. 2003, ApJ 587, L79

Frenk, C.S. et al. 2000, astro-ph/0007362

Hu, E. M., Cowie, L.L., Capak, P., McMahon, R.G., Hayashino, T. \& Komiyama, Y. 2004, AJ 127,563

Ibata, R., Chapman, S., Ferguson, A.M.N., Lewis, G., Irwin, M. \& Tanvir, N. 2005, ApJ, submitted (astro-ph/0504164)

Kanekar, N. \& Chengalur, J.N. 2003, A\&A 399, 857

Kanekar, N. et al. 2005, in preparation

Madgwick, D.S. et al. 2003, ApJ 599, 997

Naab, T. \& Ostriker, J.P. 2005, MNRAS, submitted

Nissen, P.E., Chen, Y.Q., Asplund, M. \& Pettini, M. 2004, A\&A 415, 993

Pettini, M. \& Pagel, B.E.J. 2004, MNRAS 348, L59

Reddy, N.A., Erb, D.K., Steidel, C.C., Shapley, A.E., Adelberger, K.L. \& Pettini, M. 2005, ApJ in press

Shapley, A.E., Steidel, C.C., Adelberger, K.L., Dickinson, M., Giavalisco, M. \& Pettini, M. 2001, ApJ 562, 95

Shapley, A.E., Steidel, C.C., Erb, D.K., Reddy, N.A., Adelberger, K.L., Pettini, M., Barmby, P. \& Huang, J. 2005, ApJ in press (astro-ph/0503485)

Steidel, C.C. 1993, "The Environment and Evolution of Galaxies", J.M. Shull \& H.A. Thronson eds., (Dordrecht: Kluwer Academic Publishers), 263

Steidel, C.C., Adelberger, K.L., Shapley, A.E., Pettini, M., Dickinson, M. \& Giavalisco, M. 2003, ApJ 592, 728

Steidel, C.C., Pettini, M. \& Hamilton, D. 1995, AJ 110, 2519

Steidel, C.C., Shapley, A.E., Pettini, M., Adelberger, K.L., Erb, D.K., Reddy, N.A. \& Hunt, M.P. 2004, ApJ 604, 534

Taniguchi, Y. et al. 2005, PASJ 57, 165

Tolstoy, E. 2005, ESO workshop on "Chemical Abundances and Mixing in Stars", L. Pasquini \& S. Randich eds., in press (astro-ph/0412067) 
Wolfe, A.M. 2005, Proc. IAU Coll. 199, P.R. Williams, C. Shu, \& B. Méenard eds., in press

Zwaan, M.A., van der Hulst, J.M., Briggs, F.H., Verheijen, M.A.W. \& Ryan-Weber, E.V. 2005, MNRAS, submitted

\section{Discussion}

READ: Are the kinematics of DLAs consistent with the low velocity dispersions of most dwarf ellipticals?

Pettini: The short answer is "Yes, in some cases". But interpreting the velocity structure of interstellar absorption lines in terms of the underlying gravitational potential is far from straightforward, because small parcels of gas accelerated by energetic events such as supernova explosions, can add significantly to the overall velocity spread of saturated absorption lines. Opinions differ on how much weight to place on such kinematical evidence.

SkILLMAN: Given that dEs are normally found with companion large galaxies, wouldn't the damped Ly $\alpha$ systems be more likely to be associated with a nearby $L^{*}$ galaxy?

PetTini: You would think so, wouldn't you? But it all boils down to how the crosssection for $\mathrm{H}$ I absorption scales with the galaxy luminosity. If each $L^{*}$ galaxy had many dwarf companions, and if the scaling of $\mathrm{H}$ I radius with luminosity was flatter than the Holmberg relation, then dwarf galaxies may well contribute significantly to the DLA population. This at least seems to be the case in the nearby universe, where $\mathrm{H}$ I surveys show that $87 \%$ of the DLA cross-section is in galaxies fainter than $L^{*}$, and $45 \%$ in galaxies with $L<1 / 10 L^{*}$ (Zwaan et al. 2005). The metallicity evidence I have presented is also more in line with an origin in galaxies less massive than the Milky Way. However, at this meeting we also heard from Rodrigo Ibata of the recent discovery of a very extensive disk of metal-poor stars in M31 (Ibata et al. 2005). Such a structure would match very closely the known properties of DLAs at $z=2-3$.

Conselice: Since the mass function of Lyman break galaxies is so steep, could these be the progenitors of dwarf galaxies?

Pettini: That was the gist of my presentation. Most detailed studies of Lyman break galaxies up to now have focussed on the bright end of the luminosity function. At present we know too little about sub- $L^{*}$ LBGs to make a definite connection with today's galaxy populations, although I drew attention to Ly $\alpha$ emitters and DLAs as possible progenitors of dwarf ellipticals, at least in some cases. Both LAEs and DLAs are to be found at the steep faint end of the luminosity function of high redshift galaxies. 